\title{
Neutral current coherent pion production
}

\author{
L. Alvarez-Ruso, L. S. Geng, and M. J. Vicente Vacas \\ Departamento de Física Teórica and IFIC, Universidad de Valencia - CSIC; \\ Institutos de Investigación de Paterna, Aptdo. 22085, 46071 Valencia, Spain.
}

\begin{abstract}
We investigate the neutrino induced coherent pion production reaction at low and intermediate energies. The model includes pion, nucleon and $\Delta(1232)$ as the relevant hadronic degrees of freedom. Nuclear medium effects on the production mechanisms and pion distortion are taken into account. We obtain that the dominance of the $\Delta$ excitation holds due to large cancellations among the background contributions. We consider two sets of vector and axial-vector $N-\Delta$ transition form-factors, evidencing the strong sensitivity of the results to the axial coupling $C_{5}^{A}(0)$. The differences between neutrino and antineutrino cross sections, emerging from interference terms, are also discussed.
\end{abstract}

PACS numbers: 25.30.Pt, 13.15.+g, 23.40.Bw

\section{INTRODUCTION}

The study of weak coherent pion production in charged current $(\mathrm{CC})$ and neutral current $(\mathrm{NC})$ processes, is in the agenda of several current and future experiments [1, 2, 3, 4]. For instance, the SciBooNE detector 2] should be able to identify $\pi^{0}$ 's emitted in the forward direction, where most of the coherent fraction of the total $\mathrm{NC} \pi^{0}$ production is concentrated. MINER $\nu \mathrm{A}$ [3] will collect data with high statistics, allowing for a clear separation between coherent and incoherent processes, and the comparison between neutrino and antineutrino cross sections. The measurements will be performed on $\mathrm{C}, \mathrm{Fe}$ and $\mathrm{Pb}$ targets, so that the $\mathrm{A}$ dependence can be established. The NC reaction $\nu+A \rightarrow \nu+\pi^{0}+A$ is of particular importance as a source of background in $\nu_{e}$ appearance oscillation experiments [4, 5]. The presently available data on coherent $\mathrm{NC} \pi^{0}$ production are scarce. They were obtained at $\left\langle E_{\nu}\right\rangle=2 \mathrm{GeV}$ [6] or higher [7], often with large error bars that complicate the discrimination between theoretical predictions.

Many theoretical calculations used PCAC extrapolated to $q^{2} \neq 0[8,9,10]$. Other studies focused on the neutrino-energy region around $1 \mathrm{GeV}$, where the excitation of the $\Delta(1232)$ resonance gives the dominant contribution to weak pion production on the nucleon. They have stressed the relevance of the modification of the $\Delta$ spectral function inside the nuclear medium [11, 12, 13, 14]. Another important ingredient is the pion distortion. In Refs. [8, 10], it is taken into account by factorizing the pion-nucleus elastic cross section. In a more general fashion, it can be incorporated in the amplitude by means of the distorted wave Born approximation, using a pion wave function obtained in the eikonal limit [13] or by solving the Klein-Gordon equation with a realistic optical potential [12, 14].

In this article we extend our model of Ref. [14] by adding low energy background terms to $\Delta$ excitation, and apply it to the NC case. Background terms for the weak pion production on the nucleon were considered in the past in Refs. [15], and more recently in Refs. [16, 17]. In Ref. [16] it was shown the importance of the background terms and the dressing of the $\Delta$ vertices due to the rescattering. Later, this model has been applied to incoherent pion production in nuclei [18]. In Ref. [17] the background terms were derived using the non-linear $\sigma$ model for pions and nucleons. The work of Ref. [17] and some recent lattice [19] and quark model [20] calculations suggest smaller values for the axial-vector $N-\Delta$ transition form factors $(\mathrm{FF})$ than those traditionally found in the literature $^{1}$. Therefore, we explore the sensitivity of the weak coherent pion production cross sections to different choices of $\mathrm{FF}$ in the energy range of MiniBooNE and $\mathrm{K} 2 \mathrm{~K}$ experiments.

\section{THEORETICAL MODEL}

We have applied the model of Ref. [17] for weak pion production on the nucleon to the description of the NC coherent reaction $\nu(k)+A \rightarrow \nu\left(k^{\prime}\right)+\pi^{0}\left(p_{\pi}\right)+A$ on (nearly) isospin symmetric nuclei such as ${ }^{12} \mathrm{C},{ }^{27} \mathrm{Al}$ and ${ }^{56} \mathrm{Fe}$. Besides the dominant direct $\Delta$ excitation [21], this model includes the crossed $\Delta$ term and nucleon pole terms both direct and crossed shown in Fig. 1. The ini-

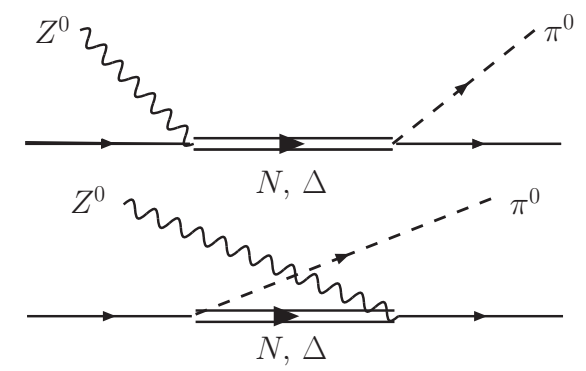

FIG. 1: Mechanisms contributing to coherent $\pi^{0}$ production in isospin symmetric matter.

\footnotetext{
${ }^{1}$ Note, however, that a direct comparison of theoretical with empirical FF is not straightforward due to the dressing of the $\Delta$ vertices as obtained in Ref. [16].
} 
tial and final nucleons are in the same quantum state as required by coherence. Other possible contributions (see Fig. 2 of Ref. [17] for the complete set of diagrams) cancel for isospin symmetric nuclei and will not be considered. The cross section for this reaction is given by

$$
\frac{d \sigma}{d \Omega_{\nu} d E_{\nu} d \Omega_{\pi}}=\frac{1}{8} \frac{\left|\vec{k}^{\prime}\right|\left|\vec{p}_{\pi}\right|}{|\vec{k}|} \frac{1}{(2 \pi)^{5}} \frac{G^{2}}{2}\left|l_{\mu} J_{A}^{\mu}\right|^{2},
$$

where the neutrino current $l_{\mu}=\bar{u}_{\nu}\left(k^{\prime}\right) \gamma_{\mu}\left(1-\gamma_{5}\right) u_{\nu}(k)$. The nuclear current $J_{A}^{\mu}$, obtained as the coherent sum over all nucleons, for all four mechanisms is

$$
\begin{aligned}
J_{\Delta d}^{\mu} & =\frac{i}{\sqrt{6}} \frac{f^{*}}{m_{\pi}} \int d^{3} r e^{i \vec{q} \cdot \vec{r}} \rho(r) F_{\Delta}\left(p_{d}\right) D_{\Delta}\left(p_{d}\right) \\
& \times \hat{p}_{\pi}^{\alpha} \operatorname{Tr}\left[\bar{u}(0) \Lambda_{\alpha \beta} \mathcal{A}^{\beta \mu} u(0)\right] \phi_{>}^{*}\left(\vec{p}_{\pi}, \vec{r}\right) \\
J_{\Delta c}^{\mu} & =\frac{i}{\sqrt{6}} \frac{f^{*}}{m_{\pi}} \int d^{3} r e^{i \vec{q} \cdot \vec{r}} \rho(r) F_{\Delta}\left(p_{c}\right) D_{\Delta}\left(p_{c}\right) \\
& \times \hat{p}_{\pi}^{\alpha} \operatorname{Tr}\left[\bar{u}(0) \tilde{\mathcal{A}}^{\beta \mu} \Lambda_{\beta \alpha} u(0)\right] \phi_{>}^{*}\left(\vec{p}_{\pi}, \vec{r}\right) \\
J_{N d}^{\mu} & =-i \frac{g_{A}}{8 f_{\pi}} \int d^{3} r e^{i \vec{q} \cdot \vec{r}} \rho(r) F_{N}\left(p_{d}\right) D_{N}\left(p_{d}\right) \\
& \times \hat{p}_{\pi}^{\alpha} \operatorname{Tr}\left[\bar{u}(0) \gamma_{\alpha} \gamma_{5}\left(\not p_{d}+m_{N}\right) \mathcal{B}_{V}^{\mu} u(0)\right] \phi_{>}^{*}\left(\vec{p}_{\pi}, \vec{r}\right), \\
J_{N c}^{\mu} & =-i \frac{g_{A}}{8 f_{\pi}} \int d^{3} r e^{i \vec{q} \cdot \vec{r}} \rho(r) F_{N}\left(p_{c}\right) D_{N}\left(p_{c}\right) \\
& \times \hat{p}_{\pi}^{\alpha} \operatorname{Tr}\left[\bar{u}(0) \mathcal{B}_{V}^{\mu}\left(\not \phi_{d}+m_{N}\right) \gamma_{\alpha} \gamma_{5} u(0)\right] \phi_{>}^{*}\left(\vec{p}_{\pi}, \vec{r}\right) .(5)
\end{aligned}
$$

Here, $g_{A}=1.26$ is the axial coupling of the nucleon, $f_{\pi}=$ $92.4 \mathrm{MeV}$, the pion decay constant and $f^{*}=2.13$, the $\Delta \rightarrow N \pi$ decay coupling; $m_{\pi}$ and $m_{N}$ stand for the pion and nucleon masses. The local nuclear density is denoted by $\rho(r) . \quad D_{\Delta}\left(p^{\prime}\right) \Lambda_{\alpha \beta}\left(p^{\prime}\right)$ and $D_{N}\left(p^{\prime}\right)\left(\not p^{\prime \prime}+m_{N}\right)$ are the $\Delta$ and nucleon propagators with the momentum of the intermediate state $p^{\prime}$ being $p_{d}=p_{i}+q=p_{f}+p_{\pi}$ for the direct diagrams, and $p_{c}=p_{i}-p_{\pi}=p_{f}-q$ for the crossed ones; $p_{i}$ and $p_{f}$ are the initial and final nucleon momenta while $q$ is the momentum transfered by the lepton $q=$ $k-k^{\prime}$. The effect of hadron internal structure on the $\Delta N \pi$ and $N N \pi$ vertices has been parametrized as [22]

$$
F_{\Delta(N)}\left(p^{\prime}\right)=\frac{\Lambda^{4}}{\Lambda^{4}+\left(p^{\prime 2}-m_{\Delta(N)}^{2}\right)^{2}},
$$

with $\Lambda=1 \mathrm{GeV}$. In the evaluation of the spin traces, we neglect corrections of the order $\left(\vec{p}_{i(f)} / m_{N}\right)$ which is equivalent to setting nucleon three-momenta to zero. On the other side, in the evaluation of $D_{\Delta(N)}\left(p^{\prime}\right)$, we assume that the three-momentum transfered to the nucleus is equally shared by the initial and final nucleons, so that $\vec{p}_{i(f)}= \pm\left(\vec{p}_{\pi}-\vec{q}\right) / 2[23,24]$.

The function $\mathcal{B}_{V}^{\mu}$ is given in terms of the vector and axial-vector nucleon form factors as follows

$$
\mathcal{B}_{V}^{\mu}=\tilde{F}_{1}^{V} \gamma^{\mu}+i \tilde{F}_{2}^{V} \sigma^{\mu \nu} \frac{q_{\nu}}{2 m_{N}}-\tilde{F}_{A}^{V} \gamma^{\mu} \gamma_{5}-\tilde{F}_{P}^{V} q^{\mu} \gamma_{5},
$$

where $\tilde{F}_{1,2}^{V}=\left(1-2 \sin ^{2} \theta_{W}\right)\left(F_{1,2}^{p}-F_{1,2}^{n}\right), \tilde{F}_{A, P}^{V}=F_{A, P}$. We use the BBA-2003 parametrization [25] for the vector
$\mathrm{FF}$, and the standard dipole $F_{A}=g_{A}\left(1-q^{2} / M_{A}^{2}\right)^{-2}$ with $M_{A}=1 \mathrm{GeV}$. PCAC has been applied to relate $F_{P}$ to $F_{A} . \quad \mathcal{A}^{\beta \mu}$ carries the information about the weak $N-\Delta$ transition $\left(\tilde{\mathcal{A}}^{\beta \mu}\left(p^{\prime}, q\right)=\gamma_{0}\left[\mathcal{A}^{\beta \mu}\left(p^{\prime},-q\right)\right]^{\dagger} \gamma_{0}\right)$

$$
\begin{array}{r}
\mathcal{A}^{\beta \mu}=\left\{\frac{\tilde{C}_{3}^{V}}{m_{N}}\left(g^{\beta \mu} \not q-q^{\beta} \gamma^{\mu}\right)+\frac{\tilde{C}_{4}^{V}}{m_{N}^{2}}\left(g^{\beta \mu} q \cdot p^{\prime}-q^{\beta} p^{\prime \mu}\right)\right. \\
\left.+\frac{\tilde{C}_{5}^{V}}{m_{N}^{2}}\left(g^{\beta \mu} q \cdot p-q^{\beta} p^{\mu}\right)\right\} \gamma_{5}+\left\{\frac{\tilde{C}_{3}^{A}}{m_{N}}\left(g^{\beta \mu} \not q-q^{\beta} \gamma^{\mu}\right)\right. \\
\left.+\frac{\tilde{C}_{4}^{A}}{m_{N}^{2}}\left(g^{\beta \mu} q \cdot p^{\prime}-q^{\beta} p^{\prime \mu}\right)+\tilde{C}_{5}^{A} g^{\beta \mu}+\frac{\tilde{C}_{6}^{A}}{m_{N}^{2}} q^{\beta} q^{\mu}\right\},
\end{array}
$$

where $\tilde{C}_{i}^{V}=\left(1-2 \sin ^{2} \theta_{W}\right) C_{i}^{V}, \tilde{C}_{i}^{A}=C_{i}^{A}$.

For the $\mathrm{FF} C_{i}^{V, A}$ we consider two different parametrizations. The first one, adopted in our recent study of $\mathrm{CC}$ coherent pion production [14], assumes the $M_{1+}$ dominance of the $N-\Delta$ electromagnetic transition [26], and the Adler model [27] for the axial FF, $C_{4}^{A}=-C_{5}^{A} / 4$, $C_{3}^{A}=0$, with

$$
C_{5}^{A}=C_{5}^{A}(0)\left(1+\frac{1.21 q^{2}}{2 \mathrm{GeV}^{2}-q^{2}}\right)\left(1-\frac{q^{2}}{M_{A \Delta}^{2}}\right)^{-2} .
$$

We take $C_{5}^{A}(0)=1.2$, in agreement with the off-diagonal Goldberger-Treiman relation [26], and $M_{A \Delta}=1.28 \mathrm{GeV}$ as extracted from BNL data [28]. $C_{6}^{A}$ is related to $C_{5}^{A}$ by PCAC.

The second set of FF is taken from Ref. [17]. In the vector sector, it adopts the parametrizations of Ref. 29] obtained from a new analysis of electron-scattering data, which allows to go beyond the $M_{1+}$ approximation. In the axial sector, the Adler model is also used, but with a different ansatz for $C_{5}^{A}$ [30]

$$
C_{5}^{A}=C_{5}^{A}(0)\left(1-\frac{q^{2}}{3 M_{A \Delta}^{2}}\right)^{-1}\left(1-\frac{q^{2}}{M_{A \Delta}^{2}}\right)^{-2}
$$

Hernandez et al. [17] fit the free parameters above to the ANL data [31] with a $\pi N$ invariant mass constraint of $W<1.4 \mathrm{GeV}$ finding $M_{A \Delta}=0.985 \pm 0.082 \mathrm{GeV}$ and $C_{5}^{A}(0)=0.867 \pm 0.075$. This value of $C_{5}^{A}(0)$ is considerably smaller than the one obtained from the off-diagonal Goldberger-Treiman relation. The coherent pion production process is more forward-peaked than the incoherent one, and filters part of the background terms. For these reasons, it is specially sensitive to $C_{5}^{A}(0)$ and, therefore, better suited to constrain its value than the incoherent reaction, provided that the experimental separation of these two contributions is possible.

The modification of the $\Delta$ properties inside the nuclei 32] causes a large reduction of the coherent pion production cross section, as shown in Refs. [13, 14]. Therefore, we take it into account for the direct $\Delta$ mechanism by adding a density dependent $\Delta$ selfenergy and modifying the free width in $D_{\Delta}\left(p_{d}\right)$, as explained in Section II B of Ref. [14]. One would also expect some in-medium corrections to the amplitudes of the other diagrams but, as 
shown below, these mechanisms have only a very small impact on the observables. Thus, we neglect further modifications which would have a negligible effect.

Finally, the operator $\hat{p}_{\pi}^{\alpha}$ in Eqs. (2-5) acts on $\phi_{>}^{*}$ as

$$
\hat{p}_{\pi} \phi_{>}^{*}\left(\vec{p}_{\pi}, \vec{r}\right)=\left(\omega_{\pi} \phi_{>}^{*}\left(\vec{p}_{\pi}, \vec{r}\right), i \vec{\nabla} \phi_{>}^{*}\left(\vec{p}_{\pi}, \vec{r}\right)\right) .
$$

$\phi_{>}^{*}\left(\vec{p}_{\pi}, \vec{r}\right)$ is the distorted wave function of the outgoing pion with asymptotic momentum $\vec{p}_{\pi}$, obtained as the solution of the Klein-Gordon equation with a microscopical optical potential based on the $\Delta$-hole model. Details can be found in Section II D of Ref. [14] and in Refs. [33, 34].

\section{RESULTS}

The integrated cross section as a function of the neutrino energy is shown in Fig. 2. The comparison between the solid and the dashed lines, both obtained with the choice of FF denoted as set I, indicates that the direct $\Delta$ excitation is the dominant mechanism. The crossed $\Delta$ term accounts for about $0.6 \%$ of the cross section at $E_{\nu}=1 \mathrm{GeV}$. The direct and crossed nucleon-pole terms are approximately six times bigger than the crossed $\Delta$, but there is a large cancellation between them, so that their sum gives less than $0.2 \%$ of the total cross section. The more realistic description of the vector form

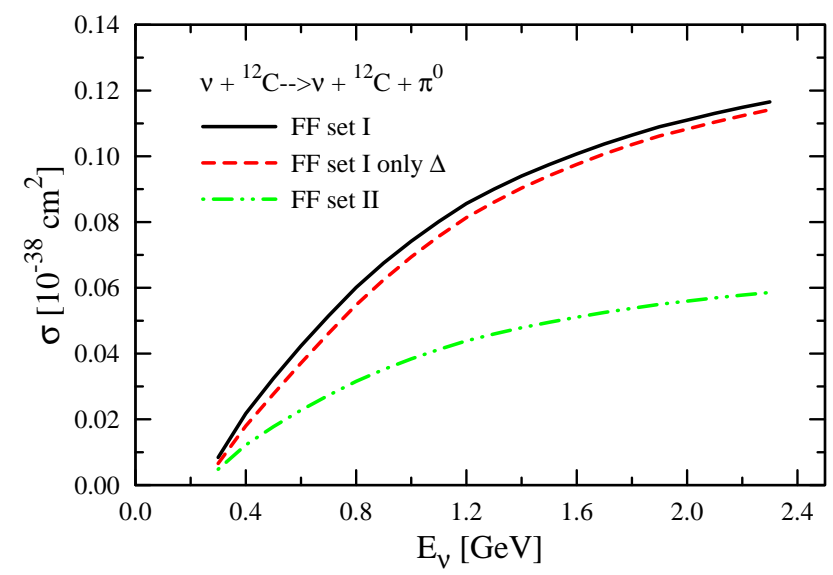

FIG. 2: (Color online) Cross section for coherent $\pi^{0}$ production on ${ }^{12} \mathrm{C}$ as a function of the neutrino energy.

factors of set II has a negligible effect on the result. On the contrary, the reduction of $C_{5}^{A}(0)$ brings down the cross section by about a factor two with respect to set I. These features can be easily understood from the fact that in the forward direction $\left(q^{2}=0\right)$, where most of the strength of this reaction is concentrated, the only form factor that contributes is $C_{5}^{A}$ [35]. Therefore, one can infer that $\sigma(\mathrm{I}) / \sigma(\mathrm{II}) \sim\left[C_{5(\mathrm{I})}^{A}(0) / C_{5(\mathrm{II})}^{A}(0)\right]^{2} \approx 1.9$.

On the basis of this argument one also expects that interference plays a minor role. It is small indeed, but not negligible, as can be observed in Fig. 3 The formalism described in the previous section is applicable to antineutrinos replacing $\left(1-\gamma_{5}\right)$ by $\left(1+\gamma_{5}\right)$ in the leptonic current. The contributions of interference terms with $q^{2} \neq 0$ are apparent in these curves, which would be otherwise identical for a given nucleus.

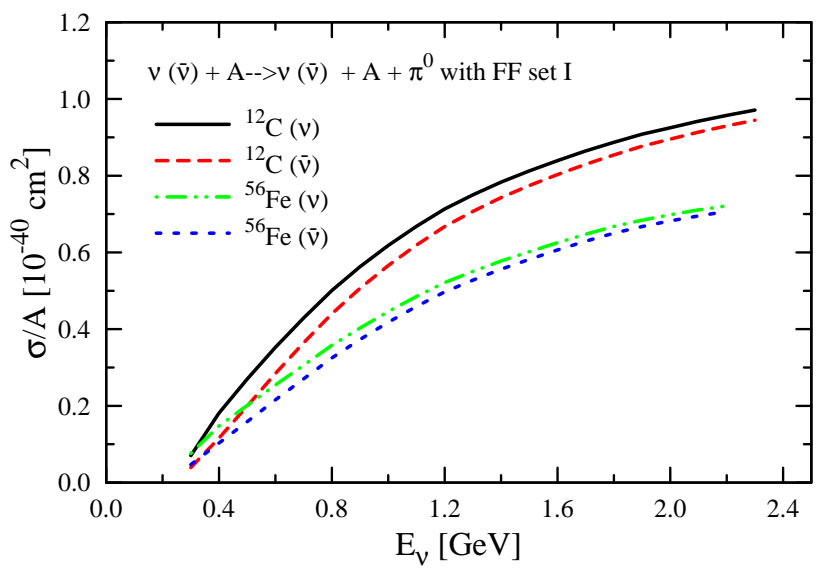

FIG. 3: (Color online) $\nu$ vs. $\bar{\nu}$ cross section for NC coherent $\pi^{0}$ production on ${ }^{12} \mathrm{C}$ and ${ }^{56} \mathrm{Fe}$ as a function of energy.

A $\sigma(\bar{\nu}) \lesssim \sigma(\nu)$ is compatible with the findings of the Aachen-Padova collaboration on ${ }^{27} \mathrm{Al}$ [6]. Our results, averaged over the CERN PS (anti)neutrino fluxes [36], for both sets I and II are shown in Table \ together with the experimental ones [ 6 ]. The results with set I are below the central experimental values but within the large error bars, while with set II the data are clearly underestimated. In any case, these theoretical values should be taken with care because these fluxes extend to high neutrino energies, where our model is less reliable.

We also show in Table \our neutrino CC cross section on ${ }^{12} \mathrm{C}$ averaged over the $\mathrm{K} 2 \mathrm{~K}$ flux [37], and the (anti) neutrino $\mathrm{NC}$ ones averaged over the corresponding MiniBooNE fluxes [38]. In the case of $\mathrm{K} 2 \mathrm{~K}$, the experimental threshold for the muon momentum $p_{\mu}>450 \mathrm{MeV} / \mathrm{c}$ is taken into account. The result with set I is slightly larger than the one given in our recent publication [14] $\left(10 \times 10^{-40} \mathrm{~cm}^{2}\right)$ due to the inclusion of background mechanisms. This number is above the upper bound obtained by K2K. While possible reasons for that discrepancy were discussed in Ref. 14], here we find that the smaller $C_{5}^{A}(0)$ value obtained in Ref. [17] (set II) is compatible with that limit. The situation is not so clear for MiniBooNE because set II predicts a very small cross section although still within the large error bars of the preliminary experimental result. In the $\bar{\nu}$ mode, the value is smaller than in the $\nu$ mode, due to both the smaller antineutrino cross sections that we obtain and the different energy distribution of the fluxes.

In summary, we have calculated the $\mathrm{NC}$ coherent pion production cross sections at low energies and compared them with the still scarce data. Our model includes all mechanisms relevant for pion production at these ener- 
TABLE I: Cross sections for weak coherent pion production in units of $10^{-40} \mathrm{~cm}^{2}$, averaged over the Aachen-Padova (AP) [36], K2K [37] and MiniBooNE [38] spectra. Columns $\sigma_{\mathrm{I}(\mathrm{II})}$ correspond to the form factor sets I and II respectively.

\begin{tabular}{lcccc}
\hline \hline Reaction & Experiment & $\sigma_{\mathrm{I}}$ & $\sigma_{\mathrm{II}}$ & $\sigma$ Exp. \\
\hline $\mathrm{NC} \nu+{ }^{27} \mathrm{Al}$ & $\mathrm{AP}$ & 19.9 & 10.1 & $29 \pm 10[6]$ \\
$\mathrm{NC} \bar{\nu}+{ }^{27} \mathrm{Al}$ & $\mathrm{AP}$ & 19.7 & 9.8 & $25 \pm 7[6]$ \\
$\mathrm{CC} \nu+{ }^{12} \mathrm{C}$ & $\mathrm{K} 2 \mathrm{~K}$ & 10.8 & 5.7 & $<7.7[1]^{a}$ \\
$\mathrm{NC} \nu+{ }^{12} \mathrm{C}$ & MiniBooNE & 5.0 & 2.6 & $7.7 \pm 1.6 \pm 3.6[39]^{b}$ \\
$\mathrm{NC} \bar{\nu}+{ }^{12} \mathrm{C}$ & MiniBooNE & 4.6 & 2.2 & - \\
\hline \hline
\end{tabular}

${ }^{a}$ Obtained using the ratio between coherent and $\sigma^{C C}$, the total $\mathrm{CC}$ cross section, and the value for $\sigma^{C C}$ of the K2K MC simulation. ${ }^{b}$ Preliminary.

gies. We find that the process is dominated by the excitation of the $\Delta$ resonance while the background terms play only a minor role. As in the CC case, nuclear effects, mainly the $\Delta$ selfenergy and the pion distortion, produce a large reduction of the cross section. The largest uncertainty of our results comes from the $N-\Delta \mathrm{FF}$. In order to estimate it we have presented results for two representative sets. Actually, we find that the coherent $\pi$ cross section is more sensitive to the $N-\Delta$ axial FF than the incoherent one. This is due to the cancellation of the isovector terms in isospin symmetric nuclei and to the forward peaked kinematics driven by the nuclear form factor. We obtain smaller $\mathrm{NC}$ coherent $\pi$ production cross sections for neutrinos than for antineutrinos. Interference terms in the production amplitudes are responsible for this difference.

\section{Acknowledgments}

We thank M. Sorel and G. Zeller for useful discussions and valuable communications. This work was partially supported by the MEC contract FIS2006-03438, the Generalitat Valenciana ACOMP07/302, and the EU Integrated Infrastructure Initiative Hadron Physics Project contract RII3-CT-2004-506078.
[1] M. Hasegawa et al. (K2K), Phys. Rev. Lett. 95, 252301 (2005); J. L. Raaf (BooNE), Nucl. Phys. Proc. Suppl. 139, 47 (2005); M. O. Wascko (MiniBooNE), Nucl. Phys. Proc. Suppl. 159, 79 (2006).

[2] K. B. M. Mahn, Nucl. Phys. Proc. Suppl. 159, 237 (2006).

[3] D. Drakoulakos et al. (Minerva) (2004), hep-ex/0405002.

[4] D. S. Ayres et al. (NOvA) (2004), hep-ex/0503053.

[5] A. A. Aguilar-Arevalo et al. (MiniBooNE) (2007), arXiv:0704.1500 [hep-ex].

[6] H. Faissner et al., Phys. Lett. B125, 230 (1983).

[7] E. Isiksal, D. Rein, and J. G. Morfin, Phys. Rev. Lett. 52, 1096 (1984); H. J. Grabosch et al. (SKAT), Zeit. Phys. C31, 203 (1986); F. Bergsma et al. (CHARM), Phys. Lett. B157, 469 (1985); M. Aderholz et al. (E632), Phys. Rev. Lett. 63, 2349 (1989); S. Willocq et al. (E632), Phys. Rev. D47, 2661 (1993).

[8] D. Rein and L. M. Sehgal, Nucl. Phys. B223, 29 (1983).

[9] A. A. Belkov and B. Z. Kopeliovich, Sov. J. Nucl. Phys. 46, 499 (1987).

[10] E. A. Paschos, A. Kartavtsev, and G. J. Gounaris, Phys. Rev. D74, 054007 (2006).

[11] H.-c. Kim, S. Schramm, and C. J. Horowitz, Phys. Rev. C53, 3131 (1996).

[12] N. G. Kelkar, E. Oset, and P. Fernandez de Cordoba, Phys. Rev. C55, 1964 (1997).

[13] S. K. Singh, M. Sajjad Athar, and S. Ahmad, Phys. Rev. Lett. 96, 241801 (2006).

[14] L. Alvarez-Ruso, L. S. Geng, S. Hirenzaki, and M. J. Vicente Vacas, Phys. Rev. C75, 055501 (2007).

[15] G. L. Fogli and G. Nardulli, Nucl. Phys. B160, 116 (1979); ibid. B165, 162 (1980).

[16] T. Sato, D. Uno, and T. S. H. Lee, Phys. Rev. C67, 065201 (2003).

[17] E. Hernandez, J. Nieves, and M. Valverde, Phys. Rev. D76, 033005 (2007).
[18] B. Szczerbinska, T. Sato, K. Kubodera, and T. S. H. Lee, Phys. Lett. B649, 132 (2007).

[19] C. Alexandrou, T. Leontiou, J. W. Negele, and A. Tsapalis, Phys. Rev. Lett. 98, 052003 (2007).

[20] D. Barquilla-Cano, A. J. Buchmann, and E. Hernandez, Phys. Rev. C75, 065203 (2007).

[21] T. Leitner, L. Alvarez-Ruso, and U. Mosel, Phys. Rev. C74, 065502 (2006).

[22] G. Penner and U. Mosel, Phys. Rev. C66, 055211 (2002).

[23] R. C. Carrasco, J. Nieves, and E. Oset, Nucl. Phys. A565, 797 (1993).

[24] D. Drechsel, L. Tiator, S. S. Kamalov, and S. N. Yang, Nucl. Phys. A660, 423 (1999).

[25] H. Budd, A. Bodek, and J. Arrington (2003), hepex/0308005.

[26] P. A. Schreiner and F. Von Hippel, Nucl. Phys. B58, 333 (1973).

[27] S. L. Adler, Ann. Phys. 50, 189 (1968).

[28] T. Kitagaki et al., Phys. Rev. D42, 1331 (1990).

[29] O. Lalakulich and E. A. Paschos, Phys. Rev. D71, 074003 (2005).

[30] E. A. Paschos, J.-Y. Yu, and M. Sakuda, Phys. Rev. D69, 014013 (2004).

[31] G. M. Radecky et al., Phys. Rev. D25, 1161 (1982).

[32] S. K. Singh, M. J. Vicente-Vacas, and E. Oset, Phys. Lett. B416, 23 (1998).

[33] C. Garcia-Recio, E. Oset, L. L. Salcedo, D. Strottman, and M. J. Lopez, Nucl. Phys. A526, 685 (1991).

[34] J. Nieves, E. Oset, and C. Garcia-Recio, Nucl. Phys. A554, 554 (1993).

[35] L. Alvarez-Ruso, S. K. Singh, and M. J. Vicente Vacas, Phys. Rev. C59, 3386 (1999).

[36] H. Faissner et al., Phys. Lett. B68, 377 (1977).

[37] M. H. Ahn et al. (K2K), Phys. Rev. D74, 072003 (2006).

[38] MiniBooNE (2007), private communication.

[39] J. L. Raaf (2005), fermilab-thesis-2005-20. 